\title{
REPERCUSSÕES SISTÊMICAS DA ICTERÍCIA OBSTRUTIVA
}

\author{
SYSTEMIC MANIFESTATIONS OF OBSTRUCTIVE JAUNDICE \\ Cláudia Carvalho Rizzo ${ }^{1}$, Orlando de Castro e Silva Jr², Ajith Kumar Sankarankutty³, \\ Luiz Alfredo G. Menegazzo4 \& Rubens Gutierrez Granato ${ }^{4}$
}

\begin{abstract}
Médica Anestesiologista'; Professor Associado²; Aluno do Curso de Pós Graduação ${ }^{3}$ do Departamento de Cirurgia, Ortopedia e Traumatologia ${ }^{1,2,3}$; Alunos do Curso de Graduação ${ }^{4}$ da Faculdade de Medicina de Ribeirão Preto da Universidade de São Paulo.

CorResPondÊnCIA: Prof. Dr. Orlando de Castro e Silva Jr. - Disciplina de Gastroenterologia do Departamento de Cirurgia, Ortopedia e Traumatologia da Faculdade de Medicina de Ribeirão Preto da Univesidade de São Paulo - Campus Universitário - CEP: 14048-900. Ribeirão Preto - SP.
\end{abstract}

RIZZO CC et al. Repercussões sistêmicas da icterícia obstrutiva. Medicina, Ribeirão Preto, 30: 173-182, abr./jun. 1997.

RESUMO: Pacientes com obstrução biliar extra-hepática apresentam particularidades que os tornam susceptiveis às alterações hemodinâmicas, cardíacas, sistêmicas e renais. Este artigo versa sobre os fatores indutores da função renal pós cirurgia descompressiva da via biliar, da instabilidade vascular, dos distúrbios da hemostasia e da ação dos sais biliares e bilirrubinas envolvidos na patogênese das complicações decorrentes da icterícia obstrutiva.

UNITERmoS: Obstrução das Vias Biliares Extra-hepática. Cirurgia. Hipotensão. Circulação Renal. Hemostasia.

\section{INTRODUÇÃO}

Pacientes com obstrução biliar extra-hepática (OBE) apresentam uma série de particularidades que os tornam susceptíveis a complicações de relevância clínica. Na icterícia obstrutiva, existe considerável risco de que pacientes com esta doença desenvolvam insuficiência renal aguda ${ }^{1}$, após a cirurgia de descompressão da via biliar, ocorrendo em aproximadamente 9\% dos casos, com índice de mortalidade de $76 \%$. As taxas de mortalidade e morbidade, associadas com procedimentos cirúrgicos da via biliar são baixas, exceto na presença de icterícia obstrutiva ${ }^{2}$, que podem chegar a 16\%. Vários fatores estão implicados na predisposição do paciente ictérico, e podem ser classificados em duas categorias: estado pré-operatório do paciente ictérico e fatores associados com a própria cirurgia abdominal. Contudo, a patogênese desta complicação ainda não está bem estabelecida.
Tabela I - Fatores que contribuem para a instalação da insuficiência renal no período pós-operatório, no paciente ictérico.

- Fatores relacionados ao paciente

- Anemia

- Grau de icterícia

- Bacteremia concomitante

- Colangite coexistente

- Níveis de creatinina

- Níveis de uréia

- Proteína - níveis de albumina

- Fatores relacionados ao ato operatório

- Medicação pré-anestésica

- Hemorragia

- Hipotensão arterial

- Agentes anestésicos

- Laparotomia 
Walker, em $1962^{3}$, foi o primeiro a reconhecer a associação entre icterícia e disfunção renal. A lesão renal que se instala em pacientes ictéricos, após cirurgia da via biliar é caracterizada por necrose tubular aguda, semelhante àquela que se segue à hipotensão ou insuficiência circulatória ${ }^{4,5}$. Sendo assim, parece que, na icterícia obstrutiva, existiriam fatores predisponentes que comprometeriam a função renal e sensibilizariam o rim durante a icterícia. Alguns autores, em estudos prospectivos, verificaram que a concentração plasmática elevada de bilirrubina é um fator de risco significante para morbimortalidade, após cirurgia da via biliar ${ }^{6 / 11}$.

Better \& Berl ${ }^{12}$ relataram que a habilidade do rim em concentrar ao máximo a urina é normal, quando a bilirrubina sérica se encontra abaixo de $8 \mathrm{mg} / 100 \mathrm{ml}$. A função renal de pacientes ictéricos pode estar comprometida de duas maneiras diferentes: 1) toxidade direta devido ao acúmulo de metabólitos hepáticos no tecido renal e, 2) indiretamente, em consequiência do prejuízo da função cardiovascular induzida pelas bilirrubinas e/ou sais biliares.

Sob circunstâncias normais, a via natural para excreção da bile e seus constituintes é através do trato biliar e sistema gastrointestinal. Entretanto, quando ocorre OBE, a fração de bilirrubina conjugada (não ligada à proteína) é filtrada pelos glomérulos, a bilirrubina não conjugada é fortemente ligada à albumina plasmática e, então, não é filtrada, exceto em concentrações plasmáticas extremamente elevadas ${ }^{13}$.

Estudos minuciosos e uma completa avaliação da função renal, em pacientes com icterícia obstrutiva e lesão hepatocelular aguda, não são realizados com grande frequiência. Dois importantes estudos realizados por Dawson ${ }^{14,15}$ enfatizaram a importância da manutenção da função renal normal durante e após a cirurgia, em pacientes ictéricos, pois a possibilidade destes pacientes desenvolverem insuficiência renal pós-operatória estava significativamente aumentada.

Estudos clínicos têm sido de extrema importância em identificar as alterações da insuficiência renal em pacientes com icterícia obstrutiva, entretanto estudos experimentais têm demonstrado sua utilidade em definir os mecanismos fisiopatológicos envolvidos. O modelo mais comumente utilizado para a icterícia obstrutiva é a ligadura do ducto biliar, em ratos, a qual acarreta diversas alterações hepáticas que podem induzir cirrose, geralmente, a partir de quatro semanas de $\mathrm{OBE}^{14}$. Outros modelos experimentais de animais, utilizados para estudo dos efeitos da icterícia, incluem a criação de "shunts" hepáticocava, infusão direta de bile, bilirrubina, ou ácidos biliares, na circulação portal ou sistêmica, e perfusão de plasma "ictérico" em rim isolado ${ }^{15 / 18}$.

Há diferenças tanto quantitativas como qualitativas dentro e entre espécies de animais, em relação à icterícia, após a ligadura do ducto biliar, por exemplo, em cães encontram-se complicações mais graves do que em ratos, como choque circulatório ou até mesmo morte ${ }^{15}$. Não se pode deixar de levar em consideração que todos os procedimentos são realizados sob anestesia, a qual, per si, pode interferir na função cardiovascular e renal ${ }^{19 / 24}$.

\section{FISIOPATOLOGIA}

Em cães, com ducto biliar ligado (sem ressecção), o pico de icterícia e lesão hepatocelular acontece até o sétimo dia pós-operatório; já, na ligadura dupla do ducto biliar com ressecção ductal, tanto a icterícia como a lesão hepatocelular são mais graves e de duração mais prolongada ${ }^{25,26}$.

As alterações renais podem ser divididas em quatro fases (Figura 1): A primeira, de curta duração, não excede a dois dias; durante este período, a ingestão de comida e de líquidos diminui, e o animal perde peso. Isto se deve tanto ao ato cirúrgico como ao aumento das bilirrubinas e/ou sais biliares. A disfunção renal caracteriza-se por aumento na taxa de filtração glomerular e do fluxo plasmático renal, acompanhado de diurese e natriurese. Todas estas alterações acontecem simultaneamente ao desenvolvimento de hipotensão. Este aumento da perfusão renal está associado a uma reatividade renovascular alterada por alguns vasodilatadores endógenos ${ }^{27}$.

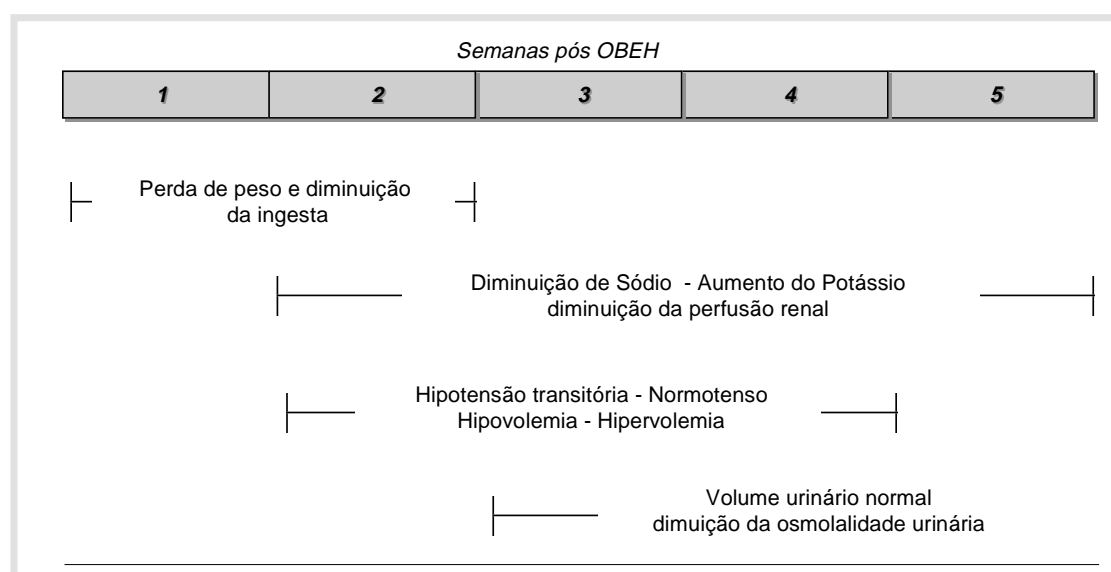

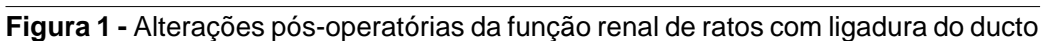
biliar. 
A segunda fase inicia-se por volta do segundo dia e vai até o fim da segunda semana. O fluxo plasmático renal e a taxa de filtração glomerular estão normais, mas a excreção de sódio começa a diminuir com o passar do tempo e a capacidade de concentrar a urina também se encontra diminuída. A hipotensão arterial, neste momento, é grave e está associada com diminuição da resposta pressórica aos estímulos da angiotensina II e da norepinefrina ${ }^{28,29}$, diminuição da função ventricular esquerda ${ }^{30}$, e respostas cronotrópica e inotrópica atenuadas ao estímulo $\beta$-adrenérgico ${ }^{31,32,33}$.

A terceira fase começa no fim da segunda semana e dura por mais duas semanas. Durante esta fase, há desenvolvimento de ascite. Hipotensão sistêmica e perda da resposta à angiotensina II e norepinefrina ainda são observadas na terceira semana pós-operatória.

A quarta fase tem início na quarta semana após a indução da OBE; além de mantidas as alterações da fase anterior, ocorre hipertensão portal, acompanhada de circulação colateral portosistêmica ${ }^{25,34}$. O quadro histopatológico do fígado lembra a cirrose e não é influenciado pelo tipo de cirurgia usado para obstruir o ducto biliar.

A explicação fisiopatológica das quatro fases é a seguinte: primeira fase - capacidade do rim em excretar metabólitos hepáticos como a bilirrubina e ácidos biliares, que estão começando a se acumular no plasma, traduzida pelo aumento da diurese e hipotensão arterial, simultaneamente, pela inibição da função tubular, que prejudica a reabsorção de sódio. A segunda fase é intermediária, nela o animal tenta manter o equilíbrio entre a necessidade de excretar os metabólitos hepáticos e a de manter a pressão arterial sistêmica normal. A terceira fase, se caracteriza por aumento da reabsorção de sódio, devido, possivelmente, a uma resposta apropriada à hipotensão sistêmica. E a quarta fase é uma extensão da terceira e ocorre como possível necessidade de compensação da hipertensão portal.

Feinberg et al. ${ }^{35}$, em estudo hemodinâmico, em cães ictéricos, mostraram que a atividade aumentada das prostaglandinas contribuía para ins- talação e manutenção de estado hemodinâmico lábil, e era revertido com o uso de indometacina (inibidor da síntese de prostaglandinas).

Este modelo experimental para indução de icterícia obstrutiva é amplamente utilizado, e as alterações hematológicas e bioquímicas diferem qualitativa e quantitativamente das observadas no cão. Nos ratos, essas alterações são dependentes do tipo de procedimento utilizado para induzir a OBE, ou seja, se o ducto biliar foi apenas ligado ou ligado e ressecado. As alterações pós-operatórias estão esquematizadas na Figura 2 e estão baseadas nas publicações relacionadas ${ }^{36 / 44}$.

No rato, após a ligadura do ducto biliar, observa-se diminuição do peso, associada à diminuição da ingestão de ração. Após três dias, observa-se variação ponderal negativa 10 a $15 \%$, independente do tipo de cirurgia realizada ${ }^{36,39,42,44}$; e diminuição da pressão arterial ${ }^{45}$. Ratos com três dias de obstrução do ducto biliar já apresentavam hipotensão arterial (Figura 3), e Bomzom et al. ${ }^{33}$ observaram que a resposta à norepinefrina, tiramina, angiotensina II e isoprenalina encontrava-se diminuída. Parece que a retração de volume intravascular poderia contribuir para a hipotensão na icterícia obstrutiva. Yarger ${ }^{44}$, Aarseth $^{36}$, Gillet ${ }^{42}$ observaram diminuição do volume sanguíneo em ratos com OBE, utilizados em seus trabalhos. Por outro lado, Better et al. $^{40}$, verificaram aumento do volume plasmático em ratos com icterícia obstrutiva.

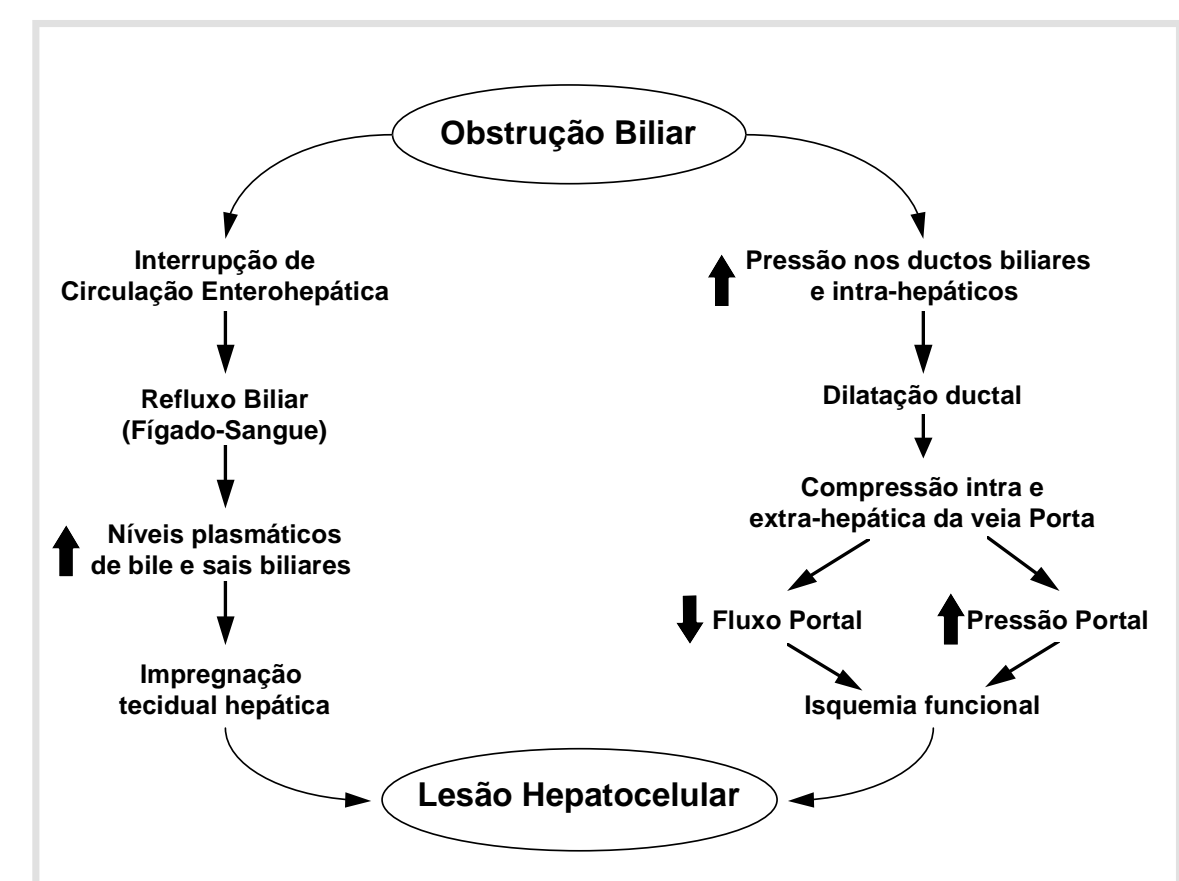

Figura 2 - Representação esquemática da fisiologia (I) na icterícia obstrutiva. 


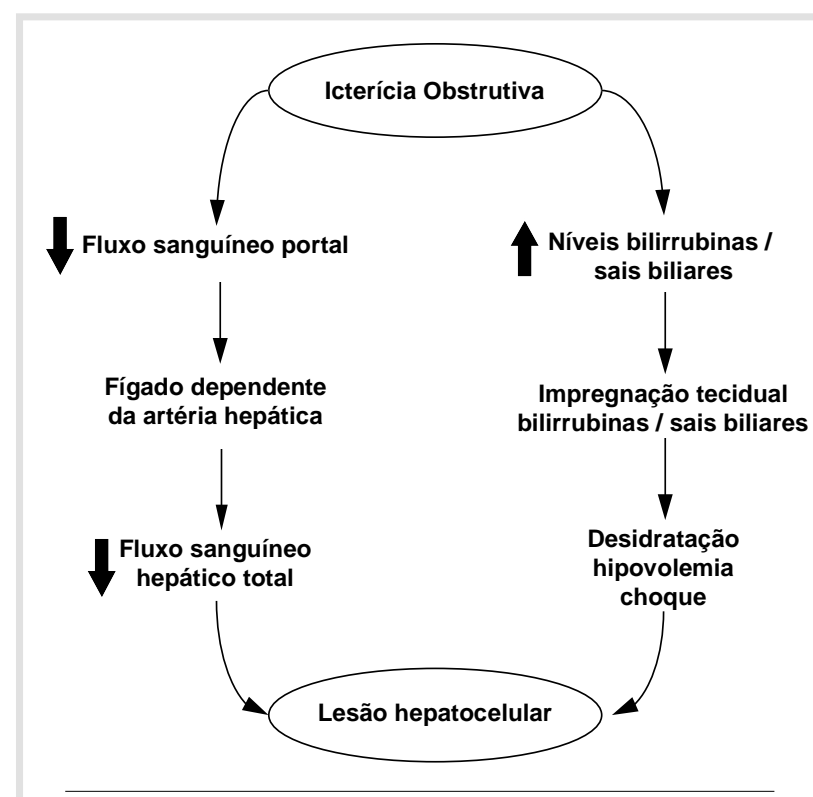

Figura 3 - Fisiopatologia (II) da icterícia obstrutiva.

A despeito desses dados conflitantes, a maioria dos investigadores relata diminuição da taxa de filltração glomerular, do fluxo plasmático renal e da excreção de sódio na fase precoce da OBE. O fluxo sanguíneo renal encontra-se reduzido, com concomitante redução na perfusão do córtex renal ${ }^{44}$, o que pode ser devido ao aumento da atividade dos receptores $\alpha$-adrenérgicos renais, que são determinantes importantes do tônus renovascular ${ }^{41}$. Esta redução na perfusão renal pode ser explicada pela queda importante da osmolalidade urinária, após vinte e quatro horas da ligadura do ducto biliar ${ }^{37}$.

A tendência à hipotensão pode ser devida a alterações vasculares periféricas ou cardíacas. Estudos, in vivo, nesses animais, revelaram resposta cronotrópica a $\beta$ agonistas prejudicada, apesar da concentração aumentada de adrenalina e noradrenalina circulante ${ }^{46}$.

Estudos mais recentes demonstraram que a icterícia em ratos, com ligadura do ducto biliar, está associada a distúrbios funcionais e metabólicos do rim e do músculo cardíaco, os quais contribuiriam para as alterações renais e hemodinâmicas observadas tanto em animais como em seres humanos ${ }^{47}$ (Figuras 4 e 5).

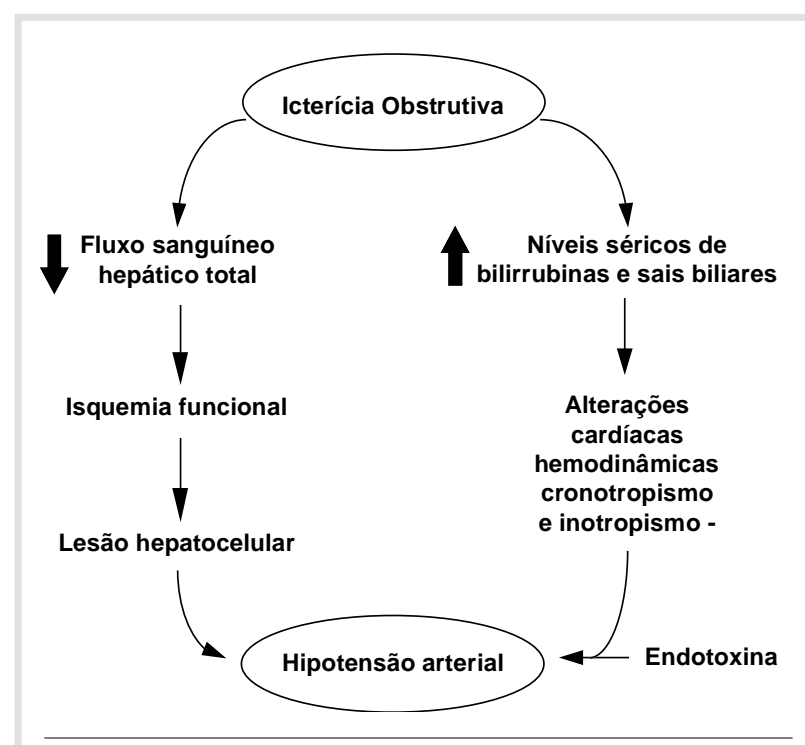

Figura 4 - Fisiopatologia (III) da Icterícia obstrutiva.

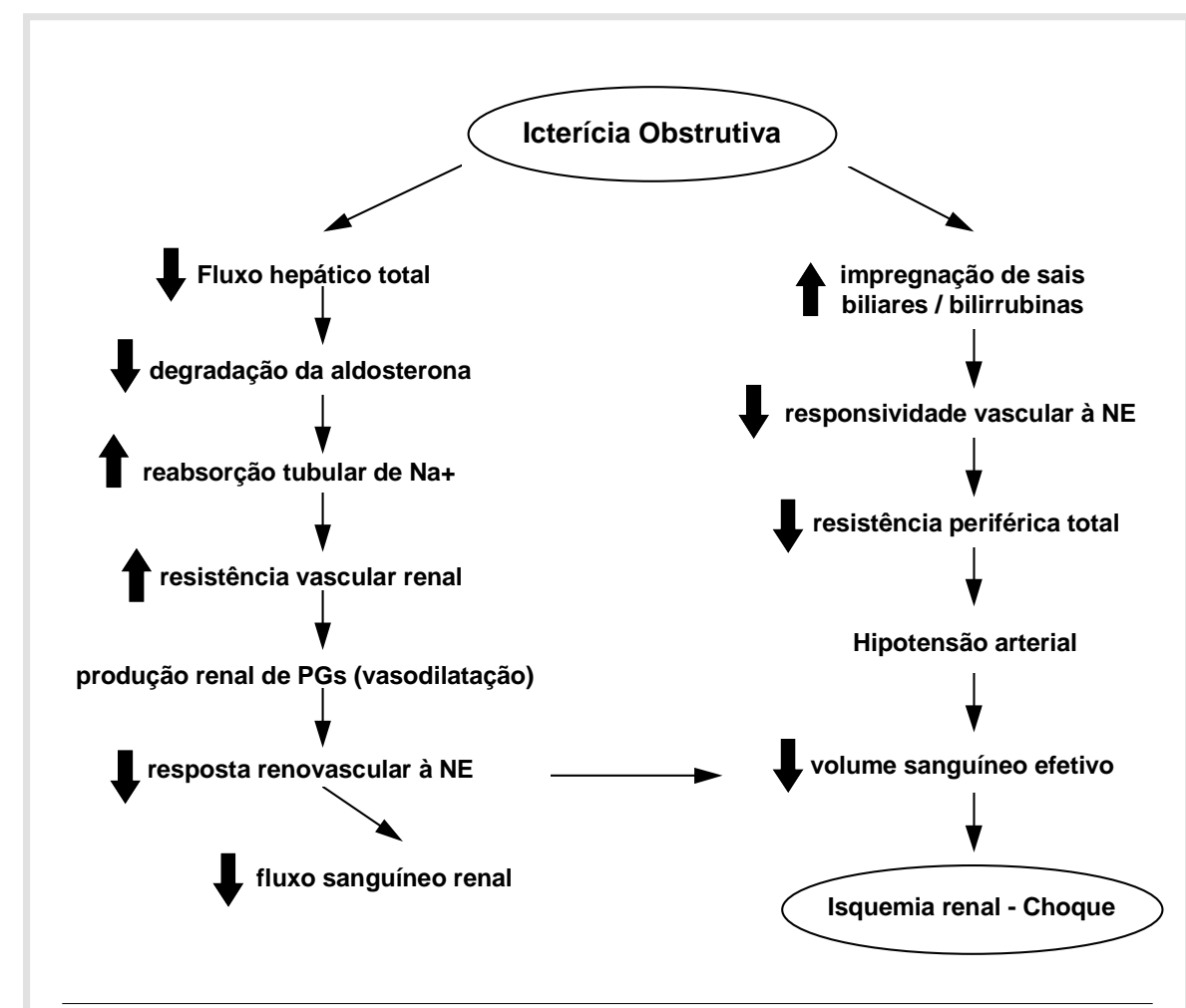

Figura 5 - Complicações renais, cardíacas e hemodinâmicas na icterícia obstrutiva. 
Como visto para o rato e para o cão, a obstrução do ducto biliar, em coelhos, também está associada à redução do peso corpóreo. As alterações verificadas também em outros animais foram hipotensão arterial sistêmica reversível, e perfusão renal diminuída ${ }^{48,49,50}$. Hishida et al. ${ }^{49}$, em estudo para avaliar a resposta da vasculatura renal à norepinefrina e angiotensina II exógenas, não encontraram alterações na reatividade renovascular em vigência de icterícia, mas mostraram que o débito cardíaco, diminuído em coelhos com icterícia obstrutiva, contribuía para a instabilidade hemodinâmica encontrada nestes animais.

Perda de peso foi encontrada em babuínos, após ligadura do ducto hepático. A icterícia decorrente da OBE está associada com a depressão de vários sistemas enzimáticos intracelulares que levam à diminuição da síntese protéica e do metabolismo dos carboidratos e das alterações hemodinâmicas sistêmicas.

Experimentos realizados nestes animais concentraram-se no estudo dos efeitos da icterícia sobre o fluxo sanguíneo renal e sua distribuição intra-renal, sendo que o principal achado foi redução, tempo-dependente, do fluxo sanguíneo renal, sem nenhuma alteração na pressão arterial sistêmica ${ }^{51}$. Esses autores observaram, ainda, que a perfusão renal diminuída estava associada ao aumento da atividade renovascular ao estímulo da norepinefrina, devido a maior atividade dos $\alpha$-adrenoreceptores renais.

\section{Aparelho Cardiovascular e icterícia obstrutiva}

Em 1932, Meakin ${ }^{52}$ descreveu as observações de paciente com hipertensão essencial que desenvolveu icterícia com obstrução biliar completa. Ele notou que, com o início da icterícia, a pressão arterial voltou aos índices pressóricos normais e, somente, retornou ao seu valor elevado, com o resolução do quadro ictérico. Este mesmo autor observou, em pacientes ictéricos, que a pressão sistólica e diastólica apresentavam tendência a serem mais baixas do que aquela observada na população normal. A bradicardia era outro achado clínico comum em pacientes ictéricos, provavelmente, devida ao efeito depressor do miocárdio pelos ácidos biliares.

Em 1956, Zollinger \& Williams ${ }^{2}$ demonstraram que pacientes ictéricos, submetidos à cirurgia da via biliar, eram susceptíveis à crise hipotensiva, após episódios de hemorragia, durante o ato operatório. Esses mesmos autores verificaram que essa susceptibilidade aumentada poderia ser atenuada através da expansão volêmica do paciente ictérico, antes da operação, o que foi confirmado também por Cattell e Birstingl ${ }^{53}$ e, recentemente, por Rizzo \& Castro e Silva $\mathrm{Jr}^{54}$.
Mesmo tendo em mente a possibilidade de ocorrência dessas alterações cardiovasculares, a hemorragia, a hipotensão arterial ou mesmo o choque, permanecem até os dias atuais como fatores etiológicos importantes na elevada taxa de morbimortalidade associada ao tratamento cirúrgico da icterícia obstrutiva ${ }^{8,55}$. O outro aspecto relacionado às complicações cardiovaculares é o de que, na vigência de icterícia obstrutiva ou mesmo hepatite infecciosa, os pacientes tendem à hipotensão arterial, com perda da resposta pressórica ou vasoconstrictora à angitensina II e à norepinefrina ${ }^{56,57}$.

Saito, em $1981^{58}$, observou que pacientes com icterícia obstrutiva, antes da desobstrução da via biliar, tinham o chamado padrão de circulação hipercinético, isto é, hipotensão arterial associada à resistência vascular periférica diminuída e débito cardíaco aumentado com freqüência cardíaca normal. Este quadro clínico é semelhante àquele observado em pacientes com hipertensão portal, por cirrose hepática.

\section{Hemodinâmica e icterícia obstrutiva}

O débito cardíaco, em animais com OBE, encontra-se, geralmente, normal ou aumentado, e a diminuição na pressão arterial deve-se à diminuição na resistência vascular periférica ${ }^{49,59,60,61}$. Cães com icterícia induzida por anastomose coledococava desenvolvem diminuição semelhante na pressão arterial, associada com aumento do débito cardíaco e diminuição da resistência vascular periférica ${ }^{15}$.

Bomzom et al. ${ }^{62}$, também, encontraram resposta vascular diminuída à norepinefrina, na musculatura esquelética. Porém, em estudo posterior, em ratos ictéricos, demonstraram que o metabolismo e a captação pulmonar de norepinefrina estavam aumentados, podendo haver diminuição dos níveis circulantes de norepinefrina ${ }^{15}$. Bomzom \& $\mathrm{Kew}^{51}$ relataram resposta diminuída à norepinefrina, medida pelas alterações no fluxo sanguíneo esquelético de babuínos ictéricos e no membro isolado perfundido do rato. Mais recentemente, achados semelhantes foram observados tanto em anéis aórticos helicoidais como na veia porta isolada ${ }^{63}$. Finberg et al. ${ }^{29}$ relataram discreta diminuição da resposta pressora à norepinefrina, em cães com OBE, entretanto, essa resposta à angiotensina II estava marcantemente diminuída. A diminuição dessa resposta poderia explicar a diminuição na resistência vascular periférica, observada em animais ictéricos.

Em resumo, os efeitos cardiovasculares e hemodinâmicos da icterícia obstrutiva, em seres humanos e em animais, são: diminuição da resistência vascular periférica com pressão arterial normal ou baixa 
(maioria dos casos), débito cardíaco elevado ou normal, e resposta hipotensora acentuada à depleção de volume. Estas alterações podem, em parte, ser secundárias a alterações na reatividade vascular.

\section{Função renal e icterícia obstrutiva}

Alterações na reatividade vascular a vários agentes vasoativos têm sido investigadas, incluindo respostas à noradrenalina. Entretanto os dados disponíveis até o momento ainda são controversos. Prostaglandinas, especialmente PGE, são conhecidas como moduladoras do fluxo sanguíneo renal ${ }^{55}$, pois têm a capacidade de diminuir a vasoconstricção renal e, com isto, manter a função e o fluxo sanguíneo renais adequados. Há evidências de que as prostaglandinas exercem papel importante na manutenção do fluxo sanguíneo renal em cirróticos ${ }^{64}$. Trabalhos recentes têm demonstrado que a produção de prostaglandinas de origem renal estão aumentadas em cães com $\mathrm{OBE}^{26}$ e, em assim sendo, as PGs ajudam a preservar a taxa de filtração glomerular e o fluxo sangüíneo renal normais, sugerindo que realmente existe aumento da atividade das prostaglandinas na vigência de icterícia.

Trabalhos na literatura demonstraram, em coelhos ictéricos, diminuição na responsividade vascular à $\mathrm{NE}$, em todo o rim, tanto na artéria renal como nos ramos intermediários. Esses autores acreditam que a atenuação da responsividade renovascular possa ser mediada pelas prostaglandinas, isto provavelmente, devido à síntese aumentada ou pela estimulação por fatores ainda não identificados e presentes no soro ictérico ${ }^{65}$.

A possibilidade de que endotoxinas possam ser responsáveis pelas complicações renais pós-operatórias pode ser devida à correlação da endotoxemia sistêmica pós-operatória e da taxa de filtração glomerular diminuída, em pacientes com OBE, submetidos a descompressão biliar ${ }^{66}$.

Em animais com OBE, níveis plasmáticos elevados de endotoxina podem reduzir a pressão arterial sistêmica e modificar a hemodinâmica renal, sendo que a endotoxina poderia ser considerada o principal fator no desenvolvimento das complicações renais e cardiovasculares na icterícia ${ }^{66 / 70}$.

\section{Ácidos biliares e bilirrubinas na patogênese das complicações da icterícia obstrutiva}

Meakin, em $1932^{52}$, mostrou, experimentalmente, que a infusão de bile e ácidos biliares, por via endovenosa, causava bradicardia com concomitante queda da pressão arterial, conseqüente à diminuição do débito cardíaco, na icterícia, obstrutiva. O acúmulo de ácidos biliares no plasma de pacientes ictéricos tem sido considerado o fator etiológico responsável pelas complicações cardiovasculares da icterícia obstrutiva. Muitos estudiosos têm se esforçado para determinar se a bile ou um de seus constituintes tem ou não efeito tóxico direto no rim. Alguns trabalhos mostraram que a bilirrubina pode desacoplar a mitocôndria durante a fosforilação oxidativa ${ }^{71}$. Ozawa et al. ${ }^{72}$ demostraram que a curva de tolerância à glicose anormal, em pacientes ictéricos, correlacionava-se com o desenvolvimento de insuficiência renal aguda pós-operatória. Esses autores postularam que a alteração da função mitocondrial, induzida pela bilirrubina, acarretava não somente diminuição da reserva hepática, mas, também, alterações da função renal. Experimentos com animais também levaram Joubert $(1978)^{73}$ à conclusão de que ácidos biliares exerciam um efeito cronotrópico negativo, mediado pelo vago, em ratos anestesiados. Dave et al. ${ }^{74}$ observaram que sais biliares, aplicados diretamente no nó sinoatrial, induziam à bradicardia em cães. Entretanto trabalhos, na literatura ${ }^{75}$, mostraram que os sais biliares, após injeção intravenosa, falharam em provocar bradicardia ou qualquer tipo de alteração no ritmo cardíaco. Outros autores também foram incapazes de demonstrar a correlação da bradicardia sinusal e níveis séricos elevados de sais biliares ${ }^{76}$. Porém Bomzom et al. ${ }^{77}$ demonstraram, in vitro, que a ligadura do ducto biliar reduzia as respostas contráteis da musculatura lisa vascular e extravascular à noradrenalina, bem como a exposição do músculo liso aos sais biliares causava redução na contratilidade vascular.

Estudos dos efeitos da bile e sais biliares, realizados no músculo cardíaco isolado, indicaram que estas substâncias têm efeito cardíaco cronotrópico e inotrópico negativo ${ }^{73,78,79,80}$, os quais se manifestam por hipotensão arterial sistêmica. Entretanto, não está estabelecido se os sais biliares exerceriam influência sobre outros fatores como diminuição da resistência periférica, resposta pressórica, atenuada aos vasoconstrictores, ou alterações do volume intravascular, que, também, regulam a pressão arterial.

Bomzom e cols., observaram, em babuínos, que concentrações sanguíneas elevadas de taurocolato de sódio não alteravam a perfusão renal, tampouco aumentavam a sensibilidade renovascular à norepinefrina $^{77}$. No cão, a infusão intra-renal de bile provocava diurese e natriurese, enquanto que a pressão arterial, taxa de filtração glomerular e o fluxo sanguíneo renal diminuíam, sem qualquer alteração na hemodinâmica renal $^{16,81,18}$. 
A bilirrubina, também, é considerada importante na patogênese das complicações da icterícia, desde que é conhecida como um composto tóxico, em altas concentrações, pelos seus efeitos deletérios na integridade anatomofuncional hepatocelular ${ }^{12,82}$. Evidências contrárias, sugerindo que a bilirrubina não estaria implicada na gênese destes fatores são demonstradas por Alon et al ${ }^{15}$, Finestone et al. ${ }^{81}$ e Naidu ${ }^{83}$.

\section{Endotoxemia}

A ocorrência de endotoxemia parece estar relacionada com o grau de icterícia obstrutiva e com a diminuição de "clearance" de creatinina, no pós-operatório ${ }^{66,84}$. O uso de endotoxina diminui o fluxo sanguíneo renal, possivelmente por estimular a liberação de catecolaminas ${ }^{85}$. A endotoxemia, na presença de icterícia obstrutiva, pode ser secundária, tanto à ausência de sais biliares no lúmen do intestino como à disfunção do sistema retículoendotelial, levando à diminuição da resistência vascular periférica por causar vasodilatação e abertura dos "shunts" arteriovenosos ${ }^{86}$.

A endotoxina do trato gastrointestinal, absorvida para circulação portal, é normalmente depurada pelo sistema retículo endotelial do fígado. Entretanto, tem sido demonstrado que, na icterícia obstrutiva, existe depressão da função do sistema retículoendotelial, e isto facilita a passagem de endotoxina para a circulação sistêmica ${ }^{87,88,89}$. A disfunção das células de Kupffer está diretamente relacionada com o desenvolvimento de endotoxemia, pois estas são responsáveis pela depuração de endotoxinas e, conseqüentemente, o prejuízo da capacidade de "clearance" destas células pode contribuir para a endotoxemia associada com colestase e suas complicações, no pós-operatório ${ }^{90}$ (Figura 6).

\section{Hemorragia}

Em pacientes não ictéricos, a hemorragia leve ou moderada pode ser bem tolerada, porém, em pacientes ictéricos, a hemorragia, durante o ato cirúrgico, inva- riavelmente é grave, e pode, per si, reduzir a pressão de perfusão renal. A combinação da anestesia, icterícia e hemorragia, maior que $10 \%$ do volume intravascular, está associada com alta taxa de mortalidade ${ }^{69} . \mathrm{Pa}-$ rece que a susceptibilidade dos animais ictéricos, aumentada ao choque hemorrágico, durante a cirurgia, tem origem extra-renal, provavelmente, devido a um déficit no volume circulante efetivo. Assim, a sobrecarga de volume intravascular melhoraria a função cardiovascular com resposta renal adequada ${ }^{60,61,91,92,93}$. Sasha et al. ${ }^{61}$ demonstraram que a sobrecarga no volume intravascular acarretava aumento do débito cardíaco, tanto em animais com OBE como em controles.

Em resumo, a insuficiência renal aguda e a instabilidade vascular ainda se constituem em graves complicações em pacientes portadores de icterícia obstrutiva. Os fatores indutores destas alterações sistêmicas foram enfatizados neste artigo.

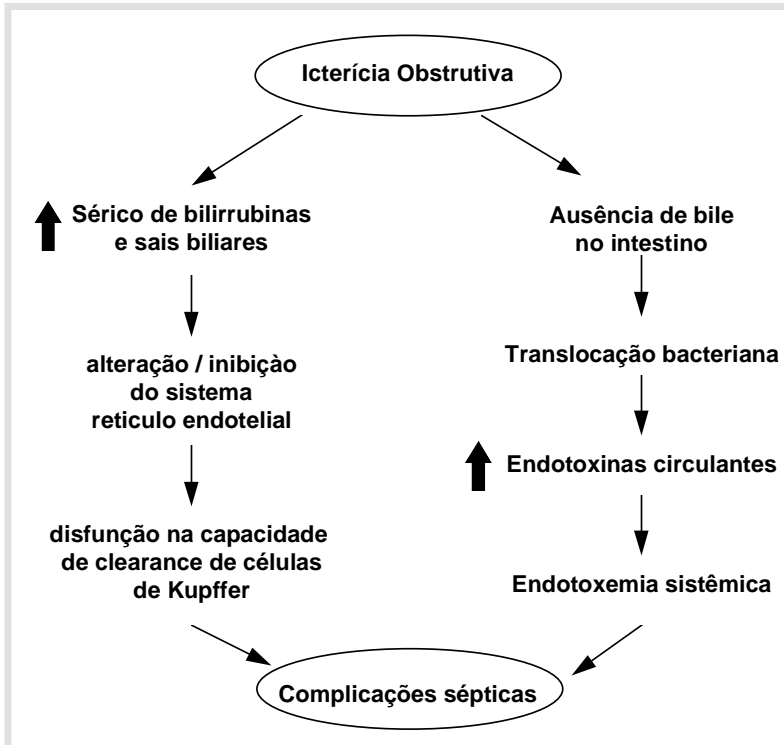

Figura 6 - Outras causas que contribuem para as complicações sépticas na icterícia obstrutiva

RIZZO CC et al. Systemic manifestations of obstructive jaundice. Medicina, Ribeirão Preto, 30: 173-182, apr./june 1997.

ABSTRACT: Patients with signs and symptoms of obstructive jaundice have particularities that make them susceptible to hemodynamic, cardiac, sistemic and renal alterations. This review reports about the inductors factor from renal function following surgery to reliese the biliary obstruction, vascular instabilityand depression of cardiovascular function due to bile acids and bilirrubin levels implicated in the pathogenesis of these compications.

UNITERMS: Hypotension. Surgery. Renal Circulation. Hemostasis. Bile Duct Obstruction, Extrahepatic. 


\section{REFERÊNCIAS BIBLIOGRÁFICAS}

1 - DAWSON JL. The incidence of postoperative renal failure in obstructive jaundice. Br J Surg 52: 663-665, 1965.

2 - ZOLLINGER RM \& WILLIAMS RD.Surgical aspects of jaundice. Surgery 39: 1016-1030, 1956.

3 - WALKER JG. Renal failure in jaundice. Proc R Soc Med 55: 570, 1962.

4 - DAWSON JL. Acute post-operative renal failure in obstructive jaundice. Ann R Coll Surg Engl 42: 163-181, 1968.

5 - LASSEN NA \& THOMSON AC. The pathogenesis of the hepatorenal syndrome. Acta Med Scand 160: 165-173, 1958.

6 - BLAMEY SL et al. Prediction of risk in biliary surgery. Br $\mathbf{J}$ Surg 70: 535-538, 1983.

7 - DAWSON JL. Post-operative renal function in obstructive jaundice: effect of manitol diuresis. Br Med J 1: 82-86, 1965.

8 - DIXON JM et al. Factors affecting morbidity and mortality after surgery for obstructive jaundice. Gut 24: 845-852, 1983.

9 - HUNT DR. Identification of risk factors and their application to the management of obstructive jaundice. Aust NZ J Surg 50: 476-480, 1980.

10 - NAKAYAMA T; IKEDA A \& OKUDA K. Percutaneous transhepatic drainage of the biliary techinique and results in 104 cases. Gastroenterology 74: 554-559, 1978.

11 - PITT HA et al. Factors affecting mortality in biliary tract surgery. Am J Surg 141: 66-72, 1980.

12 - BETTER OS \& BERL T. Jaundice and the kidney. In: SUKI WN \& EKNOYAN G eds: The kidney in systemic disease, 2th ed. John Wiley, New York, p. 521-537, 1981.

13 - ODELL GB. Studies in kernicterus. I: The protein binding of bilirrubin. J Clin Invest 38: 823-833, 1971.

14 - GLIEDSON ML et al. An experimental hepatorenal syndrome. Surg Gynecol Obstet 131: 34-40, 1970.

15 - ALON U et al. Effect of isolated cholaemia on systemic haemodynamics and kidney function in conscious dogs. Clin Sci 63: 59-64, 1982.

16 - ALON U et al. The effect of intrarenal infusion of bile on kidney function in the dog. Clin Sci 62: 431-433, 1982.

17 - TOPUZLU C \& STAHL WM. Effect of bile infusion on the dog kidney. N Engl J Med 274: 760-763, 1966.

18 - BURGER BM et al. The role of angiotensin in the canine renal vascular response to barbiturate anesthesia. Circ Res 38: 196-202, 1976.

19 - CONWAY CM \& ELLIS DB. The hemodynamic effects of short-acting barbiturates. Br J Anaesth 41: 534-542, 1969.

20 - HABIF DV et al. The renal and hepatic blood flow, glomerular filtration rate and urinary output of eletrolytes during cyclopropane, ether and thiopental anesthesia, operation and immediate postoperative period. Surgery 30: 241-245,1951.
21 - MAZZE RI et al. Renal function during anesthesia and surgery: Effects of halothane anesthesia. Anesthesiology 24: 279-284, 1963.

22 - PAPPER S et al. The effect of morphine sulphate upon the renal excretion of water and solute in man. $\mathbf{J}$ Lab Clin Med 50: 692-704, 1957.

23 - WARREN DS \& LEDINGHAM JGG. Renal circulatory responses to general anesthesia in the rabbit: studies using radioactive microspheres. Clin Sci Mol Med 48: 61-66, 1975.

24 - BOMZOM L et al. Bile salts, obstructive jaundice and renal blood flow. Isr J Med Sci 15: 169-173, 1979.

25 - SNEELL AM; GREENE CH \& ROWNTREE LG. Diseases of the liver. VII. Furtehr studies in experimental obstructive jaundice. Arch Intern Med 40: 471-487, 1927.

26 - ZAMBRASKI EJ \& DUNN MJ. Importance of renal prostaglandins in control of renal function after chronic ligation of the common bile duct in dogs. J Lab Clin Med 103: 549-559, 1984.

27 - LEVY M; FINESTONE H \& FECHNER C. Action of renal vasodilators in dogs following acute biliary obstruction. J Surg Res 36: 163-171, 1984.

28 - BLOOM D; Mc CALDEN TA \& ROSENDORF C. Effects of jaundiced plasma on vascular sensitivity to noradrenalin. Kidney Int 8: 149-157, 1975.

29 - FINBERG JPM; SYROP HA \& BETTER OS. Blunted pressor response to angiotensin and sympathomimetic amines in bileduct ligated dogs. Clin Sci 61: 535-539, 1981.

30 - GREEN J et al. The "jaundiced heart": a possible explanation for postoperative shock in obstructive jaundice. Surgery 100: 14-19,1986.

31 - BINAH OS et al. Obstructive jaundice blunts myocardial contractile response to isoprenaline in dogs: a clue to the susceptibility of jaundiced patients to shock. Clin Sci 69: 647-653, 1985.

32 - BOMZOM A et al. Modification of pulmonary metabolism of noradrenaline in experimental obstructive jaundice. Biochem Pharmacol 34: 3049-3054, 1985.

33 - BOMZOM A et al. Systemic hypotension and decreased pressor response in dogs with chronic bile-duct ligation (CBDL). Hepatology 6: 595-600, 1986.

34 - OHLISSON EG et al. Changes in portal circulation after biliary obstruction in dogs. Am J Surg 120: 16-22, 1970.

35 - FEINBERG SJ et al. Hemodynamic function in dogs with chronic obstructive jaundice: effects of radiocontrast medium. J Surg Res 49: 55-61, 1990.

36 - AARSETH P; AARSETH S \& BERGAN A. Blood volume partition after acute cholestasis in the rat. Eur Surg Res 8: 61-70, 1981.

37 - ALLISON MEM et al. Renal function in chronic obstructive jaundice: a micropuncture studies in rats. Clin Sci Mol Med 54: 649-659, 1978.

38 - AMODIO P et al. Are urinary enzymes useful markers of kidney damage in obstructive jaundice? Nephron 39: 377-381, 1985. 
39 - BANK N \& AYNEDJIAN HS. A micropuncture study of renal salt and water retention in chronic bile duct obstruction. $\mathbf{J}$ Clin Invest 55: 994-1002, 1975.

40 - BETTER OS et al. Role of antidiuretic hormone in impaired urinary dilution associated with chronic bile-duct ligation. Clin Sci 58: 493-500, 1980.

41 - BOMZOM A; BETTER OS \& BLENDIS LM. Renal alpha-1adrenoreceptors in rats with obstructive jaundice. Nephron 42: $258-261,1986$.

42 - GILLETT DJ. The effect of obstructive jaundice on blood volume in rats. J Surg Res 11: 447-449, 1971.

43 - WEIBROUM A et al. Cardiovascular function and responsiveness in experimental obstructive jaundice. Hepatology, 1987, Submitted.

44 - YARGER WE. Intrarenal mechanisms os salt retention after bile duct ligation in rats. J Clin Invest 57: 408-418, 1976.

45 - WILLIAMS RD; ELLIOT DW \& ZOLLINGER RM. The effect of hypotension in obstructive jaundice. Arch Surg 81: 334-340, 1960.

46 - GEOFFROY P et al. Impairment of chronotropic response to the beta-agonist isoproterenol in rats with portal vein stenosis or bile-duct ligation. J Hepatol 1: \& 59, 1985. Suppl.

47 - HEIDENREICH S et al. The kidney and cardiovasacular system in obstructive jaundice: functional and metabolic studies in conscious rats. Clin Sci 73: 593-599, 1987.

48 - GIOFFI WG et al. Renal vascular reactivity in jaundice. Surgery 100: 356-362, 1986.

49 - HISHIDA A et al. Mechanisms of altered renal perfusion in the early stage of obstructive jaundice. Kidney Int 17: 223-230, 1980.

50 - YAMAMOTO $\mathrm{M}$ et al. Effect of hypovolemic hypotension on plasma proteins and hepatic energy status in jaundiced rabbits. Eur Surg Res 14: 45-55, 1982.

51 - BOMZOM L \& KEW MC. Renal blood flow in experimental obstructive jaundice: In: EPSTEIN M, ed. The kidney and liver disease, 2th ed. Elsevier Science, New York, p. 313-326, 1983.

52 - MEAKIN JC. Jaundice and blood pressure. Med Clin North Am 16: 715-729,1932.

53 - CATELL WR \& BIRSTINGL MA. Blood volume and hypotension in obstructive jaundice. Br J Surg 54: 272-277, 1967.

54 - RIZZO CC \& CASTRO e SILVA Jr. O. Anestesia em paciente com icterícia obstrutiva: relato de caso. Medicina, Ribierão Preto, 27: 242-245, 1994.

55 - DUNN MJ \& ZAMBRASKI EJ. The effect on the kidney of drugs which inhibit prostaglandin synthesis. Kidney Int 18: 609-622, 1980.

56 - MORANDINI G \& SPANEDDA M. Contibuto allo studio della reattivita vascolare periferica all'angiotensina e all noradrenalina in corso di affezioni epatiche. Minerva Med 57: 2175-2180, 1966.

57 - MORANDINI G; SPANEDDA M \& SPANEDDA L. La risposta pressoria all'angiotensina e alla noradrenalina in soggetti con affezzione epatiche. Minerva Med 58: 1794-1798, 1967.
58 - SAITO S. Clinical and experimental studies on the hyperdynamic states in obstructive jaundice. J Jap Surg Soc 82: 483-497, 1981.

59 - LEVY M; WEXLER MJ \& FECHNER C. Renal perfusion in dogs with experimental hepatic cirrhosis: role of prostaglandins. Am J Physiol 245: F521-529, 1983.

60 - MELMAN A \& MASSRY SG. Role of renal vasoldilatation in the blunted natriuresis of saline infusion in dogs with chronic bile-duct ligation. J Lab Clin Med 89: 1053-1065, 1977.

61 - SASHA SM et al. Haemodynamics studies in dogs with chronic bile-duct ligation. Clin Sci Mol Med 50: 533-537, 1976.

62 - BOMZOM L; WILTEN PB \& MCCALDEN TA. Impaired skeletal muscle vasomotor response to infused noradrenaline in baboons with obstructive jaundice. Clin Sci 55:109-112, 1978.

63 - BOMZOM A et al. Reversible supression of the vascular contractile response in rats with obstructive jaundice. $\mathbf{J}$ Lab Clin Med 105: 568-572, 1985.

64 - ZISPER RD et al. Prostaglandins: Modulators of renal function and pressor resistance in chronic liver disease. J Clin Endocrinol Metab 48: 895-900, 1979

65 - HISHIDA A et al. Renal handling of salt and water in the early stage of obstructive jaundice in rabbits. Nephron 30: 368-373, 1982.

66 - BAILEY ME. Endotoxin, bile salts and renal function in obstructive jaundice. Br J Surg 63: 774- 778, 1976

67 - FISH RE \& SPITZER JA. Continuous infusion of endotoxin from an osmotic pump in the conscious, unrestrained rat: a unique model of chronic endotoxemia. Circ Shock 12: 135-149, 1984

68 - FLETCHER MS; WESTWICK J \& KAKKAR VV. Endotoxin, prostaglandins and renal fibrin deposition in obstructive jaundice. Br J Surg 69: 625-629, 1982.

69 - LEVY M \& WEXLER MJ. Subacute endotoxemia in dogs with experimental cirrhosis and ascites: effects on renal function. Can J Physiol Pharmacol 62: 673-677, 1984.

70 - WARDLE EN \& WRIGHT NA. Endotoxin and acute renal failureassociated with obstructive jaundice. Br Med J 4: $472-473,1970$

71 - ZETTERSTROM R \& ERNSTER L. Bilirrubin, an uncoupler of oxidative phsphorylation in isolated mitochondria. Nature 178: 1335-1337, 19956.

72 - OZAWA K et al. The mechanism of supression of renal function in patients and rabbits with jaundice. Surg Gynecol Obstet 149: 54-60, 1979.

73 - JOUBERT P. An in vivo investigation of the negative chronotropic effect of cholic acid in the rat. Clin Exp Pharmacol Physiol 5: 1-8, 1978

74 - DAVE $M$ et al. Some cardiovascular actions of sodium tauroglycholate. Indian J Physiol Pharmacol 18: 93-96, 1974.

75 - DUMITRESCO-MANTE HD. Action des injections intraveineuses de sels biliares sur le rhythme de pouls normal chez l'homme. CRSoc Biol (Paris) 99: 913-914, 1928. 
76 - SONG E et al. Sinus bradycardia in obstructive jaundice: correlation with total serum bile acid concentrations. S Afr Med J 64: 548-551, 1983.

77 - BOMZOM A et al. Bile salts, hypotension and obstructive jaundice. Clin Sci 67: 177-183, 1984.

78 - BINAH O et al. Effects of bile acids on ventricular muscle contraction and eletrophysiological properties:studies in rat papillary muccle and isolated ventricular myocites. Naunyn Schmiedebergs Arch Pharmacol 335: 160-165,1987.

79 - BOGIN E; BETTER OS \& HARARI I. The effect of jaundiced sera and bile salts on cultured beating rat heart cells. Experientia 39: 1307-1308, 1983.

80 - ENRIQUEZ DE SALAMANCA R et al. Negative chronotropic effect of cholic acid on isolated rat heart. Med Chir Dig 14: 585-589, 1985.

81 - FINESTONE H; FLETCHER C \& LEVY M. Effects of bile and salt bile infusions on renal functions in dogs. Can $\mathbf{J}$ Physiol Pharmacol 62: 762-768, 1984.

82 - BAUM M; STIRLING GA \& DAWSON JL. Further study into obstructive jaundice and ischemic renal damage. $\mathbf{B r}$ Med $\mathbf{J}$ 2: 229-231, 1969.

83 - NAIDU S. The effect of chronic bile duct ligation, hepatic and extrahepatic metabolites on vascular response to noradrenaline. Master's thesis, University of the Witwatersrans, Johannesburg, South Africa, 1982.

84 - CAHILL CJ; PAIN JA \& BAILEY ME. Bile salts, endotoxin and renal function in obstructive jaundice. Br J Surg 165: 519-522, 1987.
85 - CAVANAGH D et al. Pathophysiology of endotoxin shock in the primate. Am J Obstet Gynecol 108: 705-722, 1970.

86 - LIEHR $\mathrm{H}$ et al. Endotoxin-induced liver necrosis and intravascular coagulation in rats enhanced by portacaval collateral circulation. Gut 16: 429-436, 1975.

87 - STEVENSON DK et al. Pulmonary excretion rate of carbon monoxide as an index of total bilirrubin formation in adult male wistar rats with common bile duct ligation. J Pediatr Gastroenterol 3: 790-794, 1984.

88 - KATZ S et al. Impaired bacterial clearance and trapping in obstructive jaundice. Ann Surg 199: 14-20, 1984.

89 - MORI K; MATSUMOTO K \& GANS H. On the in vivo clearance and detoxification of endotoxin by lung and liver. Ann Surg 177: 159-163, 1973.

90 - PAIN JA et al. Reticuloendothelial system phagocytic function in obstructive jaundice and its modification by a muramyl dipeptide analogue. Eur Surg Res 19: 16-22, 1987.

91 - CLEMENTS WDB et al. Effects of extrahepatic obstructive jaundice on kupffer cell clearance capacity. Arch Surg 128: 200-204, 1993.

92 - BETTER OS \& MASSRY SG. Effect of chronic bile-duct obstruction on renal handling of salt and water. J Clin Invest 51: 402-411, 1972.

93 - CHAIMOVITZ $C$ et al. Effect of renal denervation and alpha-adrenergic blockade on sodium excretion in dogs with chronic ligation of the common bile duct. Proc Soc Exp Biol Med 146: 764-770, 1974. 